\title{
Recomendando Objetos de Aprendizagem a partir das hashtags postadas no Moodle
}

\author{
Francisco Adelton Alves Ribeiro ${ }^{1}$, Luis Carlos Costa Fonseca ${ }^{2}$, Miguel de Sousa Freitas ${ }^{3}$ \\ ${ }^{1}$ Instituto Federal de Educação Ciência e Tecnologia do Maranhão (IFMA) - Codó, \\ MA - Brasil \\ ${ }^{2}$ Universidade Estadual do Maranhão (UEMA) - São Luis, MA - Brasil \\ ${ }^{3}$ Secretaria de Educação do Maranhão (SEDUC) - Caxias, MA - Brasil \\ adelton@ifma.edu.br, lccfonseca@gmail.com, qdmisofre@gmail.com
}

\begin{abstract}
The research aims to develop an additional module to the virtual learning environment - VLE Moodle, from the set of hashtags assigned in forums posted using the technique of assigning weights to known documents in the field of information retrieval - IR, allowing the execution an algorithm to classification and ranking contents from the terms quoted. This approach is based on studies of systems development as well as in social media resources. During the study, we could realize that the recommendation system is very useful not only to reduce the dispersion of users, but also to improve the interaction between them and the contents taught.
\end{abstract}

\begin{abstract}
Resumo: A pesquisa tem como objetivo o desenvolvimento de um módulo adicional ao Ambiente Virtual de Aprendizagem - AVA Moodle, a partir do conjunto de hashtags atribuídas nas postagens dos fóruns, empregando a técnica de atribuição de pesos a documentos conhecida na área da Recuperação de Informação - IR, permitindo a execução de um algoritmo para classificação e ranqueamento de conteúdos a partir dos termos mais citados. Esta abordagem baseia-se nos estudos sobre desenvolvimento de sistemas e recursos de mídias sociais. Verifica-se no decorrer da pesquisa, que o Sistema de Recomendação - SR reduz a dispersão dos usuários, melhorando a interação com os conteúdos ministrados.
\end{abstract}

\section{Introdução}

A Internet é a grande biblioteca do mundo moderno, que vem crescendo de forma exponencial nos últimos anos. Segundo pesquisa do IBOPE (2013), o total de pessoas com acesso à internet no Brasil, no primeiro trimestre de 2013, chegou a 102,3 milhões. Representando o crescimento de $9 \%$ sobre os 94,2 milhões divulgados no terceiro trimestre de 2012, fato que caracteriza o aumento de usuários e de conteúdos. Apesar do grande volume de informações disponível na Internet, muitas vezes as pessoas não conseguem encontrar uma determinada informação, ou levam muito tempo para localizá-la. 
Atualmente buscar e sugerir informações relacionadas a sugestão de conteúdos para alunos, tornou-se uma tarefa pouco trivial, este problema se torna mais crítico quando a busca envolve atividades relacionadas no âmbito da Educação a Distância $\mathrm{EaD}$, pois a pesquisa deve ser realizada a partir dos AVAs, que são softwares gerenciadores de cursos à distancia.

Diante da crescente interação social via internet, a qual tem atingido a escola e seus agentes, não se pode ignorar a influência e a necessidade de uso das Tecnologias da Informação e Comunicação - TIC como suporte efetivo ao processo de ensino/aprendizagem, uma vez que cresce o uso das redes sociais como meio de circulação das informações e nos diálogos entre pessoas, modificando-lhes comportamentos, hábitos, valores e atitudes nas suas relações.

O objetivo desse artigo é apresentar um sistema para recomendação de OA aos alunos do AVA Moodle, baseado no conjunto das hashtags atribuídas nas postagens dos fóruns, fazendo uso do esquema da frequência de ocorrência do termo no documento e do inverso da frequência do termo entre documentos da coleção.

\section{Metodologia}

A estrutura desse artigo está organizado em seções: na 2 e 3 serão apresentadas a metodologia e o referencial teórico utilizado; na 4 trata-se da solução proposta, descrevendo o algoritmo do sistema de recomendação; a seção 5 faz uma breve análise dos resultados e contribuições e, finalmente, na última, a 6 traz a conclusão.

Objetivando criar e integrar um módulo que recomende objetos de aprendizagem aos usuários do AVA Moodle, foi desenvolvido um sistema que permite localizar o conjunto de hashtags mais postadas nos fóruns da plataforma, classificando-as, ranqueando-as e sugerindo conteúdos relacionados ao tema em estudo. Atendendo aos procedimentos metodológicos foram criadas, nesse estudo, as seguintes etapas:

1. Pesquisa bibliográfica sobre sistema de recomendação, objetos de aprendizagem, etiquetagem, folksonomia e ambiente virtual de aprendizagem;

2. Utilização da técnica de atribuição de pesos a documentos utilizados na área da Recuperação da Informação - RI;

3. Adequação do esquema a frequência de ocorrência do termo no documento (Term Frequency - TF) e do inverso da frequência do termo entre documentos da coleção (Inverse Document Frequency - IDF) à estrutura dos cursos do AVA Moodle, fazendo uso de hashtags;

4. Modelar e integrar a arquitetura do sistema de recomendação ao AVA Moodle;

5. Realização de teste com 30 alunos, o que possibilitou o agrupamento das hashtags postadas nos fóruns, fornecendo ao sistema informações necessárias para recomendar OA.

Desenvolvida essas etapas, criou-se um curso no AVA Moodle, no qual os alunos inseriram hashtags em suas postagens, de forma a criar um banco de informações relevantes ao sistema, recomendando objetos de aprendizagem relacionados as postagens mais ranqueadas. 


\section{Referencial teórico}

Para melhor entender como a pesquisa foi realizada, fez-se necessário percorrer por alguns conceitos que apresentaremos uma discussão entorno destes, tendo como base os sistemas de recomendação, objetos de aprendizagem, etiquetagem, folksonomia e AVA.

\subsection{Sistema de Recomendação}

Sistemas de recomendação são agentes de informação personalizados que fornecem recomendações e sugestões de itens que possam ser de utilidade para o usuário, [Burke, 2007].

Segundo [Cazella et al., 2012], os Sistemas de Recomendação vêm auxiliar o usuário no processo de seleção de conteúdo. Em um sistema típico de filtragem de informação, as pessoas fornecem recomendações como entradas e o sistema agrega e direciona para os indivíduos que são considerados potenciais interessados.

Para [Adomavicius \& Tuzhilin, 2005] o problema da relevância nos SR pode ser formulado da seguinte forma: considerando que " $C$ " seja o conjunto de todos os usuários e " $S$ " o conjunto de todos os itens possíveis de serem recomendados - como músicas, livros, filmes, etc. A quantidade de "S" (possíveis itens a serem recomendados) pode ser muito grande, referindo-se a milhares de centenas ou milhões de itens em alguns modelos de aplicações, tais como em sistemas de recomendação de músicas e textos.

De forma semelhante, a quantidade de usuários pode também ser muito extensa, dezenas ou centenas de milhões em alguns casos. Consideremos " $u$ " a utilidade ou relevância da informação em uma função matemática que mensura a relevância do item " $s$ " para o usuário $c$, ou seja, $u: C \times S \rightarrow R$, onde $R$ é o conjunto total de recomendações ordenadas. Então, para cada usuário $c \in C$ o sistema deve escolher o item $s^{\prime} \in S$ que maximize a relevância da recomendação ao usuário. Em termos formais temos:

\section{Equação 1: cálculo da relevância dos resultados de busca nos SR}

$$
\forall c \in C, s^{\prime}{ }_{c}=\arg \max _{s \in S} u(c, s) \text {. }
$$

A função de utilidade é normalmente representada pelo ordenamento crescente obedecendo ao ranking (ranqueamento) que indica a probabilidade de um usuário específico gostar ou se interessar por um item em particular. Pode, por exemplo, ser representada por uma nota que evidencie quanto um usuário gostou de um item, exemplificando, o filme "A Rede Social" foi avaliado por um usuário como nota $8 \mathrm{em}$ um escala ranqueada de 1 a 10.

Sistemas de Recomendação são de particular importância em ambientes sociais, onde os usuários compartilham acesso a um conjunto comum de recursos. A variabilidade das características do usuário, como sua origem, seus interesses especiais e seu grau de especialização, para sugerir itens interessantes, úteis e compreensíveis para um usuário específico.

Neste estudo, usaremos a técnica de atribuição de pesos a documentos conhecidos na área de RI, ou seja, o esquema da frequência de ocorrência do termo no documento (TF), que conta quantas vezes o termo aparece e nos dá uma medida de quão bem o termo descreve o conteúdo do documento, e o inverso da frequência do termo entre documentos da coleção (IDF), que quantifica um termo como fator discriminatório para todos os documentos da coleção, para localizar o conjunto de hashtags postadas 
nos fóruns do AVA Moodle, ordenando e recomendando objetos de aprendizagem a seus utilizadores.

\subsection{Objetos de Aprendizagem}

Diante do atual contexto escolar brasileiro, os educadores necessitam de alternativas pedagógicas que auxiliem o processo de ensino/aprendizagem de forma mais eficiente [Almeida, 2001].

Com advento da Web, surgem mais possibilidades para colaboração e o compartilhamento de recursos didáticos, como os objetos de aprendizagem representam qualquer recurso usado como ferramenta pedagógica inserido em um ambiente de aprendizagem, apresentando características como: reusabilidade, adaptabilidade, granularidade, acessibilidade, durabilidade e interoperabilidade [Silva, 2010].

De acordo com a norma Standard for Learning Object Metadata (IEEE) 1484 (2002): um objeto de aprendizagem é definido como qualquer entidade, digital ou não, que possa ser usada para a aprendizagem, educação ou treinamento. Por exemplo, fotos ou imagens digitais, animações, simulações e pequenas aplicações, páginas da Web que combinam texto, imagens e outros meios ou aplicações. As principais vantagens são as possibilidades de reutilização em aulas virtuais, de adaptação de um objeto, a ilustração de conceitos abstratos e o aspecto interativo, resultando no processo ensino/aprendizagem de forma mais significativa.

Dessa forma, a evolução tecnológica tem propiciado a criação de ambientes interativos de aprendizagem, possibilitando descobertas entre os limites do saber e do aprender, impulsionado professores e educadores a repensarem seus papéis, através de reflexões sobre questões, que contribuam para apropriação e construção de novos conhecimentos, favorecendo um redimensionamento da prática pedagógica. Nesse contexto, faremos uso de um sistema de busca para localizar o conjunto de hashtags postadas nos fóruns do AVA Moodle, classificando e recomendando automaticamente conteúdos relacionados ao tema de interesse dos discentes.

\subsection{Etiquetagem e Folksonomia}

O processo de etiquetagem no mundo real é amplamente utilizado como uma maneira de rotular, identificar, localizar produtos, serviços e conteúdos, proporcionando o desenvolvimento econômico e social entre os mais diversos países e suas respectivas culturas. Diante desse amplo uso, o modelo de etiquetagem foi levado para o mundo virtual, onde a internet através da Web 2.0 apropriou-se para localizar, classificar e organizar itens de interesses do usuário. Seguindo essa tendência, o presente estudo faz uso de etiquetas para localização de informações relacionados a temática de interesse dos usuários, propiciando a aprendizagem colaborativa, cooperativa e a construção do conhecimento de modo rápido, com o propósito de motivar o pesquisador.

Etiquetagem (em inglês, tagging) é uma forma de indexação em que as próprias pessoas, no caso, os usuários da informação, classificam os documentos. A marcação de conteúdo com termos descritivos, também chamados palavras-chave ou etiquetas (em inglês tagging), é uma forma comum de organizar conteúdo para futura navegação, filtragem ou busca. Embora, essa forma de organizar conteúdo eletrônico não seja algo novo, uma forma colaborativa desse processo que foi chamada de "etiquetagem", está ganhando popularidade na Web [Golder e Huberman, 2006, p.198, tradução nossa]. 
Sendo assim, repositórios de documentos ou bibliotecas digitais, muitas vezes permitem que documentos e suas coleções sejam organizadas pelas palavras-chaves.

No AVA os usuários anotam estas palavras-chaves nas distintas ferramentas de comunicação, como os fóruns, chat, diário de bordo, etc. Essas anotações podem ser utilizadas pelo SR de modo a disponibilizar OA aos usuários, ou simplesmente para classificar conteúdos. Este processo de etiquetagem no mundo virtual é denominado de folksonomia, que é largamente utilizada em redes sociais, haja vista o twitter, facebook, Google+, entre outras. Normalmente são termos acompanhados de algum "caractere coringa", por exemplo, o "\#”.

Nesse sentido, etiquetar é uma das ações relacionadas à classificação mais incorporada ao dia a dia das pessoas. Está nos rótulos dos produtos, nos alimentos e em uma variedade de objetos de uso pessoal ou coletivo, e contém informações importantes sobre determinado objeto. Na "sociedade 2.0", etiquetar configura ação de disseminação da informação, utilizando-se da folksonomia para a atribuição de termos para representação do conhecimento.

A folksonomia resulta da junção das palavras folk (= povo: significa a classificação das coisas, em especial a informação; é definida por pessoas e para pessoas) + taxonomy (= taxonomia: termo de origem grega que significa "a ciência de classificar as coisas"), por isso a folksonomia cria relações entre coisas objetivando a categorização e a classificação de termos na Web [Catarino e Batista, 2007].

\subsection{Ambiente Virtual de Aprendizagem}

Os AVAs são sistemas computacionais disponíveis na internet, destinados ao suporte de atividades mediadas pelas TIC, que permitem integrar múltiplas mídias, ordenar informações, desenvolver interações entre pessoas e objetos de conhecimento. Tornar o AVA um ambiente mais humanizado deve ser uma meta constante em todo projeto de $\mathrm{EaD}$, seja através do uso das mídias sociais, de outras ferramentas da Internet ou mesmo de qualquer estratégia que esteja ao alcance dos tutores e dos professores no ambiente virtual [Coelho, 2012].

Os AVAs podem ser empregados como suporte para sistemas de educação a distância realizados exclusivamente on-line, para apoio às atividades presenciais de sala de aula, permitindo expandir as interações da aula para além do espaço-tempo do encontro face a face ou para suporte à atividades de formação semipresencial nas quais o ambiente virtual poderá ser utilizado tanto nas ações presenciais como nas atividades à distância.

Atualmente existem vários AVAs que podem ser utilizados pelas Instituições de Ensino em seus cursos na modalidade à distância, alguns são softwares livres, o que possibilita sua customização e acréscimo de novas funcionalidades a outros proprietários. Para contextualização desta pesquisa usamos o AVA Moodle.

\subsubsection{O Moodle}

O nome Moodle é o acrônimo de "Modular Object Oriented Dynamic Learning Environment" que pode ser traduzido por Ambiente Dinâmico de Aprendizagem Orientado por Objetos Modulares, foi desenvolvido pelo australiano Martin Dougiamas, no final dos anos 90, objetivando ofertar cursos não presenciais auxiliados pela rede mundial de computadores. Em virtude da facilidade de manuseio e controle das ações 
desenvolvidas por seus usuários, o Moodle vem sendo utilizado por inúmeros países subdesenvolvidos e em desenvolvimento. Tal fato ganha mais importância em função de ser um ambiente gratuito com código aberto ("opensource"), baseado na Web e desenvolvido atendendo aos princípios pedagógicos.

A plataforma Moodle está notavelmente associada à educação superior, no entanto há outras áreas - como o da educação corporativa - em que o Moodle é utilizado como plataforma de aprendizagem de forma ampla e customizada para atender às mais diferentes necessidades [Nunes et al., 2012].

Apesar do Moodle auxiliar no processo de ensino/aprendizagem, o mesmo apresenta algumas dificuldades, pois é um software no qual limita professores e alunos ao armazenamento por vezes estático de informações de ensino, tornando o processo de aprendizagem passivo sem que exista uma interação online ativa entre docentes e discentes [Corradi, 2009]. Objetivando melhorias e ampliação dos recursos do Moodle, a fim de auxiliar as estratégias de ensino, com novas formas de comunicação, exercício da interatividade na aprendizagem, trabalho cooperativo e na construção do conhecimento, este artigo propõe a criação de um módulo recomendador de OA para o AVA Moodle, sugerindo aos alunos conteúdos relevante baseados nas hashtags postadas nos fóruns da plataforma.

\section{Solução proposta}

Tendo em vista, as dificuldades expostas, observou-se a necessidade de desenvolver um módulo contendo sistema para recomendação de objetos de aprendizagem e integrá-lo ao AVA Moodle, mostrado na figura abaixo, sob forma de um bloco, o qual será discutido ao longo dessa seção.

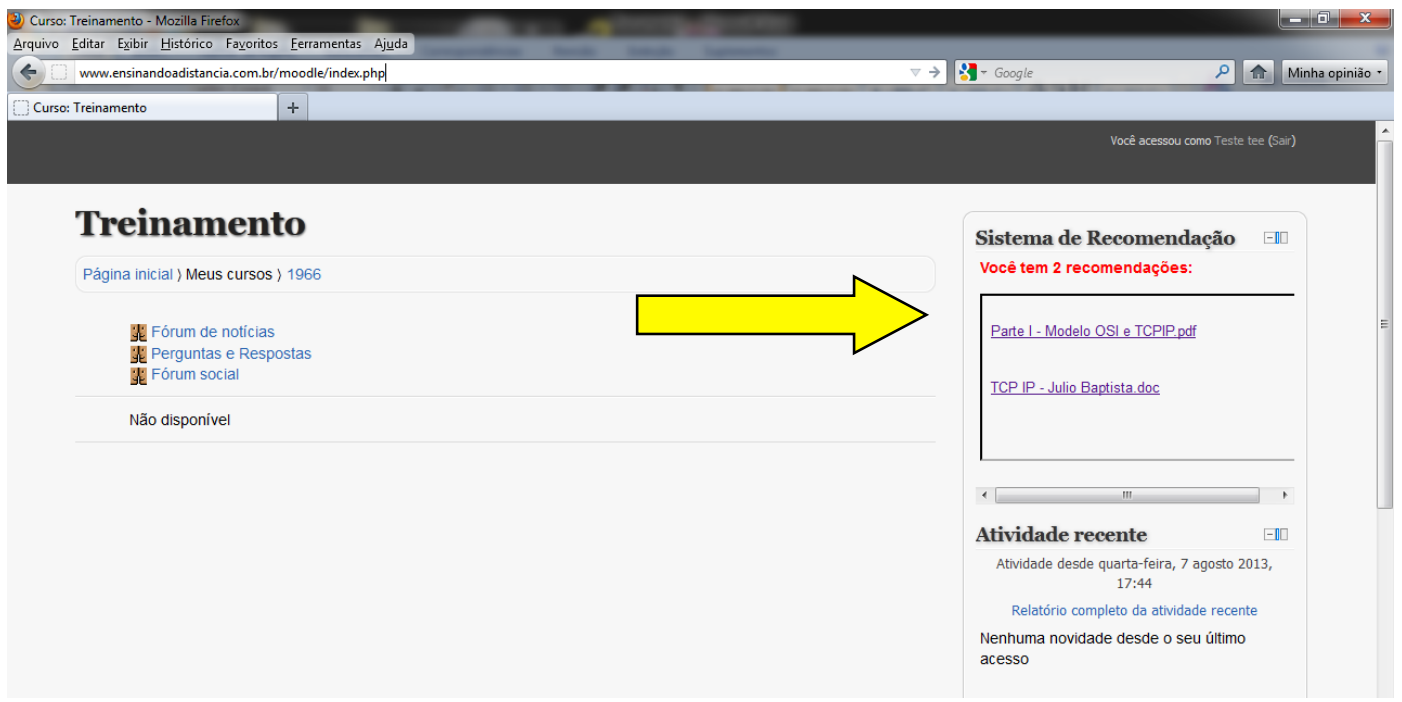

Figura 1 - Sistema de Recomendação.

Usando os fóruns do AVA Moodle, os usuários atribuirão hashtags em suas postagens, cuja finalidade é classificatória, criando agrupamentos textuais, que serão utilizadas pelo sistema para fazer as recomendações.

Essa classificação ganha relevância quando se visualiza cada hashtag como um agregado de postagens, formando assim, uma base de dados composta de recortes desconexos em torno de um mesmo conteúdo. 
O problema desta abordagem é definir como as informações serão tratadas e comparadas com os documentos contidos em repositório. Para tanto, será criado para cada hashtag $h_{j}$ um vetor de termos e pesos, contendo todos os termos das postagens de um determinado usuário que esteja marcado $\operatorname{com} h_{j}$. Para esta formulação, utilizar-se á uma técnica de atribuição de pesos a documentos conhecida na área de RI que é o esquema a frequência de ocorrência do termo no documento (TF) e o inverso da frequência do termo entre documentos da coleção (IDF). Desta forma, para cada termo (palavra) das postagens associadas a uma hashtag $h_{j}$, teremos o seguinte esquema de cálculo dos pesos $W_{i j}$ :

$$
w_{i, j}=\left\{\begin{array}{rr}
\left(1+\log f_{i, j}\right) \times \log \frac{N}{n_{i}} \text { se } f_{i, j}>0 \\
0, \text { se } f_{i, j} \leq 0
\end{array}\right.
$$

As variáveis da fórmula são detalhadas abaixo:

- $W_{i j}$ é o peso de cada termo $k_{i}$ associado à uma hashtag $h_{j}$;

- $f_{i j}$ é a frequência de ocorrência da um termo $k_{i}$ nas postagens de $h_{j}$;

- $\quad N$ é o número total de hashtags utilizadas em uma determinada sala de aula; e

- $n_{i}$ é o número de hashtags (vistas como agrupamento de posts de um usuário) que fazem referência ao termo $k_{i}$ nos fóruns de uma determinada sala de aula, disciplina ou curso (esta terminologia varia conforme a estrutura de cursos adotada);

Convém salientar que este estudo faz adequação do tradicional esquema de ranking denominado TF-IDF [Baeza-Yates e Ribeiro-Neto, 1999; Manning et al., 2009], à estrutura dos cursos do Moodle. No esquema original, a variável $N$ é o número de documentos de uma coleção e $n_{i}$ é a quantidade de documentos nos quais o termo $k_{i}$ ocorre.

Desta forma, é possível comparar a estrutura de dados das postagens (hashtags) com uma coleção de documentos em repositório. A princípio, as hashtags de melhor ranqueamento, serão aferidas com os documentos existentes no repositório, de modo a calcular suas similaridades. Para realizar o cálculo fazemos uso do Modelo Vetorial, conforme a fórmula abaixo:

$$
\operatorname{sim}\left(h_{j}, d\right)=\frac{\sum_{i=1}^{t} w_{i, j} x w_{i, d}}{\sqrt{\sum_{i=1}^{t} w_{i, j}^{2}} x \sqrt{\sum_{j=1}^{t} w_{i, d}^{2}}}
$$

Para a fórmula acima se observa uma nova variável $d$, que representa os itens que potencialmente podem ser recomendados a um usuário. Estes itens são ranqueados de acordo com o seu grau de similaridade com alguma hashtag $h_{j}$. Portanto, torna-se possível recomendar os mais variados documentos de um repositório, sejam planilhas eletrônicas, arquivos de textos, apresentações, arquivos PDF, etc, bastando apenas que eles contenham alguma informação textual que seja indexável.

A figura 2, é um diagrama de componentes o qual ilustra a arquitetura atual do sistema de recomendação, bem como sua forma de interação com o Moodle. 


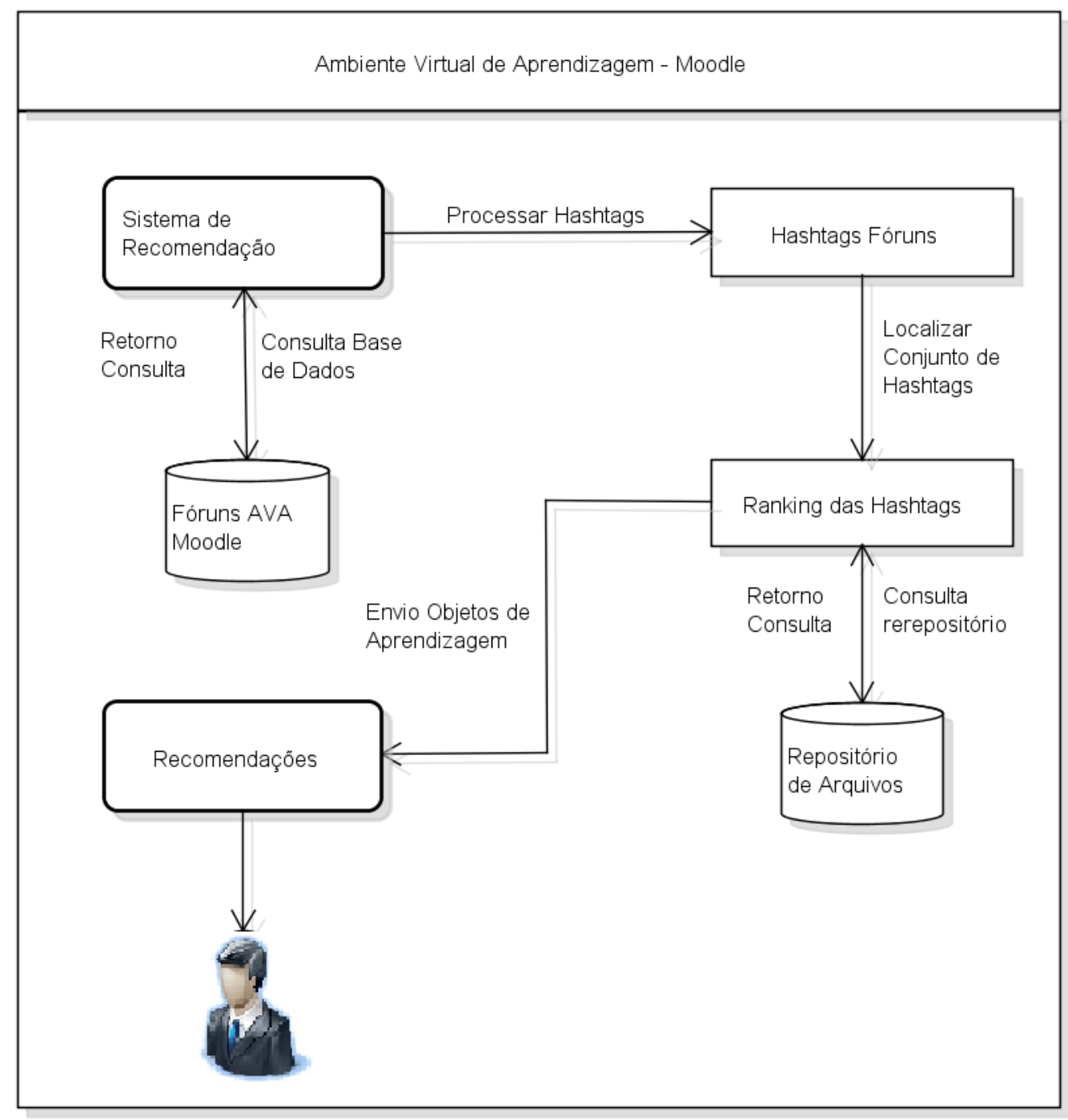

Figura 2 - Diagrama dos componentes do sistema.

\section{Fonte: Autores. 2013}

O sistema proposto é baseado na técnica de recomendação de conteúdos, no qual o algoritmo recebe consultas textuais, para extrair todas as hashtags presentes nos fóruns do AVA Moodle de forma idêntica ao processo de indexação, retornando, então, a lista de OA do repositório que possuem nomes relacionados com as hashtags. Para cada OA retornado, é calculado um escore entre o OA e a consulta através do somatório de pesos dos termos gerais via esquema vetorial TF-IDF. Finalizando o processo, os OA recuperados são ordenados através dos pesos vetoriais calculados e normalizados em relação ao maior peso encontrado no repositório.

Este sistema tem como objetivo automatizar a busca de OA, facilitando a utilização dos atores envolvidos (professor, alunos, direção, etc) na localização de conteúdos relevantes contidos no repositório de documentos do AVA Moodle. Este formato ainda pode ser modificado adotando o modelo de metabusca, onde o sistema de recomendação não mais filtraria OA de um repositório de documentos do AVA Moodle e sim submeteria a um motor de busca (Google, Yahoo, Bing, Feb, CESTA) palavraschave "hashtags" baseadas nas postagens dos alunos. 


\section{Resultados e contribuições}

Como mecanismo de avaliação preliminar, foi criado um curso denominado "Introdução à Informática" no qual foi adicionado o bloco contendo o sistema. Para realização dos teste, 30 alunos da empresa Compuservis Informática, foram convidados a participar e inserir hashtags em suas postagens nos fóruns, pelo período de 7 dias.

A avaliação foi realizada de forma quantitativa e qualitativa, na qual foi perguntado aos usuários como eles avaliarão as recomendações feitas pela ferramenta. Desta forma acredita-se que há evidências suficientes para aprimorar as recomendações.

Antes do início dos testes os alunos receberam uma ficha de avaliação contendo as instruções de acesso à plataforma e de utilização de hashtags nos fóruns do curso. Foi questionado aos usuários qual sua opinião sobre as recomendações recebidas, objetivando avaliar aspectos como relevância e layout do bloco, e se gostariam de sugerir alguma contribuição para o sistema desenvolvido.

Através da utilização do ambiente, pelos cursistas, e das respostas obtidas em fichas de avaliação, foi possível verificar a boa aceitação das recomendações, e a aprovação do layout do bloco.

O processo avaliativo apresentou limitações devido a exiguidade temporal da disponibilização do curso. Esta limitação não permitiu avaliar de forma considerável a evolução das recomendações em decorrência da limitação do tempo de uso. Porém, a fim de avaliar o funcionamento do sistema, relegando o aspecto pedagógico, a etapa de testes foi eficiente.

As recomendações ocorrerão a contento, pois os testes revelaram o pleno funcionamento do sistema, bem como sua possível utilização em larga escala, a fim de avaliar o seu caráter pedagógico. As técnicas utilizadas pelo sistema mostraram-se eficientes, apresentando recomendações padronizadas em caráter diversificado, segundo interesse do usuário.

\section{Conclusão}

A EaD vem sendo a cada dia mais utilizada pelas instituições de ensino, tanto para apoio aos cursos presenciais como para cursos a distância. Nesse cenário, uma ferramenta muito importante é o AVA. No decorrer da pesquisa percebemos que os alunos do AVA Moodle, fazem uso da internet em busca de conteúdos complementares aos seus estudos e, em muitos casos, dispersam-se navegando na grande rede, distanciando-se de seus objetivos.

Diante disso, o sistema proposto busca informação relevante para o usuário e recomenda objetos de aprendizagem, melhorando a interação dos alunos com o ambiente virtual de aprendizagem, reduzindo a dispersão e possibilitando melhoria no processo ensino/aprendizagem.

O resultado avaliativo mostrou que o sistema em fase de operação e teste, é de grande utilidade frente a outros sistemas já usados, haja vista que os objetos de aprendizagem recomendados foram avaliados em sua maioria como interessantes, pois o sistema tem sugerido conteúdos satisfatórios, dessa maneira consegue mantê-los por mais tempo na plataforma. 


\section{Referências}

Adomavicius, G., \& Tuzhilin, A. (2005). Toward the next generation of Recommender Systems: A Survey of the State-of-Art and Possible Extension. IEEE Transaction on Knowledge and Data Engeneering, vol. 17, n.6 (june), p. 734-748.

Almeida, M. E. B. Educação projetos tecnologia e conhecimento. 1. Ed. São Paulo: PROEM, 2001.

Baeza-Yates, R. A.; Ribeiro-Neto, B. Modern informationre trieval. Addison-Wesley Longman Publishing Co., Inc., 1999.

Burke, R. Hybrid Web Recommender Systems. In Brusilovsky, P., Kobsa, A., Nejdl, W. (eds.). The Adaptive Web: Methods and Strategies of Web Personalization., Lecture Notes in Computer Science, Vol. 4321, pp. 377-408, Springer, Berlin-Heidelberg, 2007.

Catarino, M. E.; Baptista, A. A. Folksonomia: um novo conceito para a organização dos recursos digitais na Web. Revista Data Grama Zero: Revista de Ciência da Informação, v.8, n.3, jun. 2007. Disponível em: <http://www.dgz.org.br/jun07/F_I_art.htm> Acesso em: 13 mar. 2012.

Cazella, S. C.; Bhear, P.; Schneider, D.; Silva, K. K.; Freitas, R. (2012), “Desenvolvendo um Sistema de Recomendação de Objetos de Aprendizagem baseado em Competências para a Educação: relato de experiências". In Anais do XXIII Simpósio Brasileiro de Informática na Educação, SBIE, Rio de Janeiro, RJ.

Coelho, G. W.; (2012), "Uso dos recursos de mídias sociais na Educação a Distância: impactos na percepção da presença social”. In Anais do XXIII Simpósio Brasileiro de Informática na Educação, SBIE, Rio de Janeiro, RJ.

Corradi, B. S. Avaliação de novas tecnologias da informação e da comunicação no processo de ensino $\begin{array}{lllll}\text { aprendizagem em uma disciplina experimental da UFV. } & 2009 .\end{array}$ http://www.tede.ufv.br/tedesimplificado/tde_arquivos/39/TDE-2010-02-08T102543Z2141/Publico/texto\%20completo.pdf>. Acesso em: 2012.

Golder, S.; Huberman, B.A. Usage patterns of collaborative tagging Systems. Journal of Information Science, v. 32, n. 2, p. 198-208, 2006.

IBOPE - Instituto Brasileiro de Opinião Pública e Estatística. Disponível em: http://www.appbrasil.org.br/ibope/free/acesso_internet.pdf. Acesso em: -24/09/2013.

IEEE Standard for Learning Object Metadata (IEEE Stda 1484.12.1, 2002). Nova York. IEEE, 2002;

Manning, C.; Raghavan, p.; Schutze, H. Introduction to information retrieval.Cambridge University Press, 2009.

Murray, J. H. Hamlet no Holodeck: O futuro da narrativa no ciberespaço. EditoraUnesp, 2003.

Nunes, C. S.; Torres, M. K. L.; Oliveira, P. C.; Nakayama, M. K. (2012) "O ambiente virtual de aprendizagem Moodle: recursos para os processos de Aprendizagem Organizacional”, In Anais do XXIII Simpósio Brasileiro de Informática na Educação, SBIE, Rio de Janeiro, RJ.

SILVA. Kézia Natália Castro. Objetos de Aprendizagem. UFF:2010 em $<$ http://www.webartigos.com/artigos/a-producao-de-material-pedagogico-a-partirdo-uso-dastic/53010/\#ixzz2Ak1AZkAJ>. Acesso em 25 de março de 2013. 